\title{
A novel microspectrometer technology for IR spectral imaging applications
}

\author{
by K. K. M. B. D. Silva*, J. Antoszewski*, T. Nguyen*, A. J. Keating*, J. M. Dell*, and L. Faraone*
}

*School of Electrical Electronic and Computer Engineering, The University of Western Australia, M018, 35 Stirling Highway Crawley, 6009, Western Australia, lorenzo.faraone@uwa.edu.au

\begin{abstract}
Including multi/hyper spectral capabilities into existing infrared imaging systems is of great significance, for military as well as civilian applications. While infrared spectral imaging systems presently exist, the spectrometer component is usually made of bulk-optics, which require macroscopic motion and, at times, require high voltages to tune the wavelength. As a result, their utility, particularly in airborne applications, is hindered by the bulk, cost, high computational load, and high power consumption.

Our work aims to address the above limitations by implementing the entire spectrometer, at the focal-plane level, using microelectromechanical systems (MEMS) technologies. This approach has several advantages over existing schemes including, reduction of cost and bulk of the system, significantly reduced voltage and power requirements, and enabling the intelligent acquisition of only data relevant to the application. We report on our microspectrometer technology, based on Fabry Perot tunable filters, compatible with large format infrared focal plane arrays, with potential for implementation as a spectrometer array at the focal plane. Our technology is inherently low voltage, which makes it compatible with focal plane array (FPA) readout circuits. Single device and small array configurations have already been demonstrated, with the array forms targeted towards multispectral and hyperspectral imaging applications. We have also demonstrated linear variable filter array technologies suited to application in process control in moving production line environments.
\end{abstract}

\section{Introduction}

State-of-the-art infrared (IR) focal plane array technologies aim to improve the performance of IR imaging systems by reducing cooling requirements and/or adding so-called multi-colour capability, which allows on-pixel information to be gathered from two or more spectral regions. Spectral information allows improved target recognition and reduced false alarm rates in military applications, and accurate temperature determination in civilian applications. It has been recognised, however, that in order to extract spectroscopic or detailed information from a cluttered image, significantly better spectral resolution is required than can be afforded by such multi-colour systems. A number of research programs are aiming to address this issue by obtaining significantly better spectral resolution by developing technologies for the integration of individual tuneable optical filters on each pixel of a detector array.

The approach chosen by The Microelectronics Research Group (MRG) is to develop a micro-electromechanical systems (MEMS) technology that is compatible with large format two-dimensional infrared focal plane arrays. Such a device structure consists of an electrostatically controlled Fabry-Perot filter that is integrated optically ahead of the individual detectors in an array. The demonstration of this technology has involved major advances in the deposition of silicon nitride $\left(\mathrm{SiN}_{\mathrm{X}}\right)$ thin films supports [1], and the development of novel MEMS actuation schemes structures for extending spectral measurement range [2].

\section{Principle and implementation of the microspectrometer}

The MRG microspectrometer, as illustrated in Fig 1(a) and (b), consists of a MEMS tunable Fabry Perot filter mounted optically ahead of an infrared photodetector. Each mirror in the microspectrometer is a multi-layer Bragg mirror structure consisting of three layers, germanium - silicon monoxide - germanium. A structural layer of silicon nitride supports the top mirror from above. This structural layer extends to the support arms that actuate the mirror structure. This fabrication process is horizontally scalable, making it compatible with implementation on a per-pixel level, at the focal plane, as illustrated conceptually in Fig. 1(c).

Metal electrodes are deposited on the bottom mirror, outside the optically active area, and on the actuation arms of the microspectrometer. Electrostatic actuation is achieved by application of a voltage between the bottom mirror and the actuation arms. This allows the top mirror to be moved, downwards in Fig. 1(a), decreasing the inter-mirror spacing and, thereby, shifting the pass-band of the spectrometer. A drawback common to many electrostatically actuated MEMS devices is the effect of dielectric charging. Dielectric charging is the gradual migration and build up of electric charge in the dielectric layer(s) between the electrodes of the MEMS structure [4]. These charging effects arise in MEMS 
primarily due to the use of insulating (dielectric) materials, in MEMS manufacture, which inherently exhibit charging behaviour. As a result of the dielectric charging, hysteresis can manifest and, generally, will cause the device response to drift with time. Dielectric charging has also been shown to be the limiting factor on lifetime of some MEMS devices [5].

These charging effects potentially become a problem when trying to drive the MEMS using a large amplitude DC analogue signal, as in the case of the microspectrometer. The resulting drift prevents accurate placement of the MEMS by the drive voltage. It is helpful, however, that these charging effects take place on a slow timescale. As charge drifts in proportion to the applied field, these charges will not move in response to a high frequency AC field. Additionally, the response of MEMS actuators to an applied actuation voltage is proportional to the square of the voltage, independent of the direction of the actuation voltage. Therefore, dielectric charging can be mitigated by the use of a high-frequency AC drive signal, at a frequency where neither the MEMS nor the dielectric charge migration will respond [6]. The MEMS will then only respond to the RMS value of the AC drive signal.

(a)

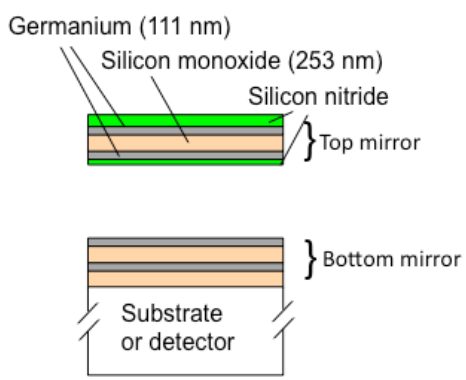

(b)

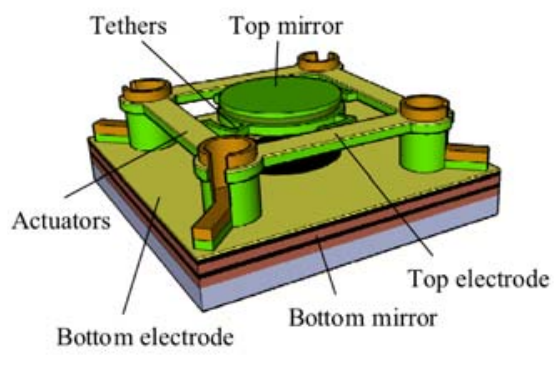

(c)

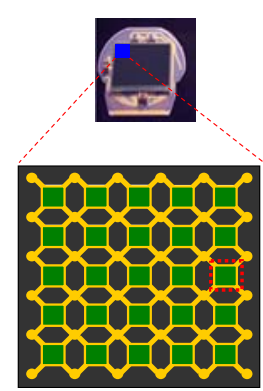

Fig 1. (a) Cross section of optical and structural layers of MRG microspectrometer, (b) $3 D$ view of micromachined Fabry-Perot filter, and (c) potential array application of the technology

Three key issues affect the performance characteristic of the microspectrometer: managing stress in the top mirror, creating tensile but low internal-stress $\mathrm{SiN}_{\mathrm{X}}$ supports, and snap-down. All three issues have to be addressed on the path to realization of a functional MEMS microspectrometer.

Stress in the top mirror arises due to a number of reasons; including (1) the fact that it is an asymmetric multilayer structure fabricated at temperatures different that of operation and (2) the fact that the top mirror is deposited on an organic material, which is subsequently removed. The result of this stress is a bowing in the top mirror. Fig. 2(a) depicts the effect of top mirror bowing on the optical performance of the microspectrometer device. In optical terms, this bowing causes a broadening of the spectral FWHM (i.e. degraded spectral resolution), as well as degradation in the peak transmission of the filter pass-band. From Fig. 2(a), it is clear that a bowing of $\delta d=100 \mathrm{~nm}$ over the $100 \mu \mathrm{m}$ mirror clearly results in very poor filter performance. On the other hand, a bowing of $15 \mathrm{~nm}$ causes only a minor degradation in performance. Hence, our stress balancing effort aimed to achieve a maximum mirror bowing of $\delta d= \pm 15 \mathrm{~nm}$. Stress balancing and flattening of the top mirror was achieved in a two-step process. Firstly, a stress-balancing layer of thin, compressive, $\mathrm{SiN}_{\mathrm{X}}$ is placed on the mirror. Secondly, an oxygen plasma ash is used, post-fabrication, to induce a second order curvature reduction in the mirror [3]. Using this technique, as seen in Fig. 2(b), we have successfully fabricated mirror structures with as little as $\pm 15 \mathrm{~nm}$ of bowing over the entire $100 \mu \mathrm{m}$ mirror, allowing near-optimal optical performance of the MEMS filter.

The significance of internal stress in the $\mathrm{SiN}_{\mathrm{x}}$ supports is in terms of device actuation. The $\mathrm{SiN}$ membranes need to be tensile to produce a flat membrane. However, if the supports are excessively tensile, large voltages are needed to actuate the top mirror, meaning that the actuation voltage becomes incompatible with the low-voltage readout/control circuit of the focal plane array. High quality $\mathrm{SiN}_{\mathrm{x}}$ films require optimization of a multi-parameter space including temperature, gas flow ratios, pressure and RF power. Extensive studies of $\mathrm{SiN}_{\mathrm{x}}$ deposition in a PECVD system has devised a set of conditions that can achieve structures with appropriate mechanical properties and low internal stress which allows for significant reduction of actuation voltage. At the process conditions chosen, the Young's Modulus and hardness of our films are typically $100 \pm 10 \mathrm{GPa}$ and $8 \pm 2 \mathrm{GPa}$, respectively. As can be seen in Fig. 3(b), the actuation voltages for these MEMS filters are less than $25 \mathrm{~V}$.

Snap-down occurs during electrostatic actuating of MEMS structures, because the electrostatic attraction force scales with the inverse of the electrode separation, while the mechanical restoring force only scales in proportion to the displacement of the electrode. If the top mirror were to be actuated directly, by applying a voltage between the top mirror and the bottom electrode directly below, when the mirror has displaced $1 / 3$ of the initial gap between the mirrors, the mechanical restoring force can no longer overcome the electrostatic actuation force. The system then becomes unstable, and the top mirror collapses ("snaps down") to the bottom electrode. We have implemented a novel MEMS structure, 
where the actuation happens on the arms that provide the restoring mechanical spring force to the mirror. Due to this modification, and by doubly-supporting these beams by two posts, we are able to take advantage of the non-linear behaviour of the mechanical spring constant of the structure. The non-linearity in the spring constant shifts the instability point, of this electro-mechanical system, to beyond $50 \%$ of the initial gap.

(a)

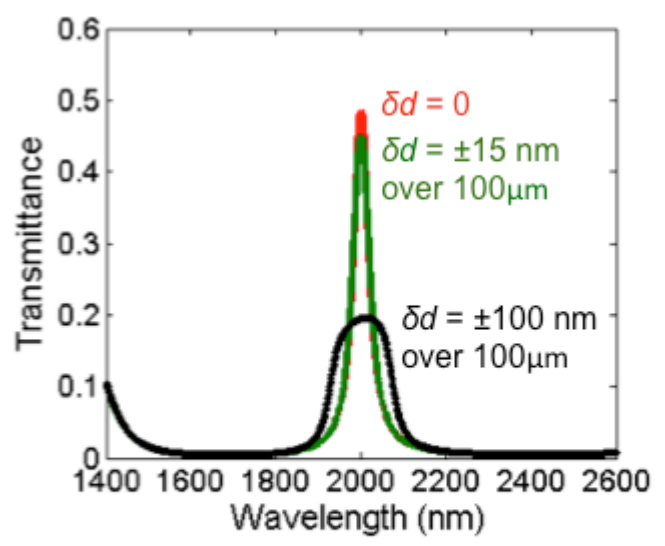

(b)

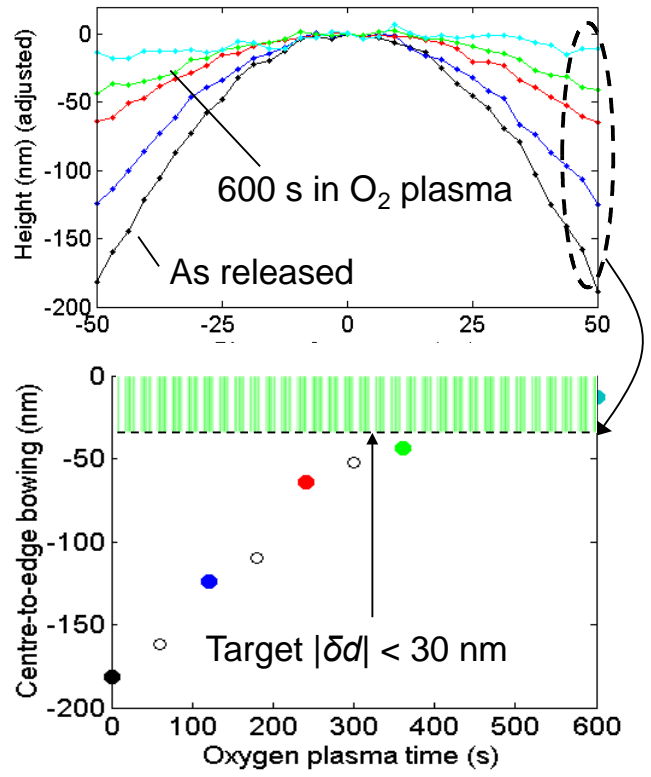

Fig 2. (a) Effect of various degrees of bowing ( $\delta d)$ across the entire $100 \mu \mathrm{m}$ mirror area, and (b) mirror flattening using an oxygen plasma

With this design, we have demonstrated over 50\% gap tuning. Fig. 3(a) demonstrates this design has allowed the shortwave infrared microspectrometer to scan over a $900 \mathrm{~nm}$ spectral range, with an actuation voltage of less than $25 \mathrm{~V}$. Similarly, we have demonstrated a spectral scan range of $900 \mathrm{~nm}$ in the midwave infrared, with an actuation voltage of less than 20 V. Fig. 3(b) shows the measured tuning curve, of the shortwave IR devices, against the modelled performance. The circular points on Fig. 3(b) indicate optically measured position measurements, while the diamonds indicate spectral peak locations. The solid line is the theoretically predicted curve. It is clear that both measurements, agree well with the predictions from the analytical model.

(a)

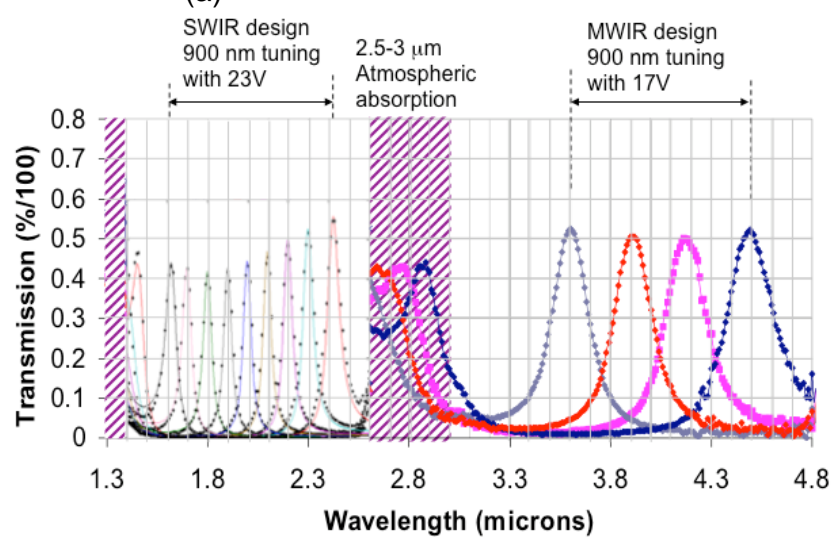

(b)

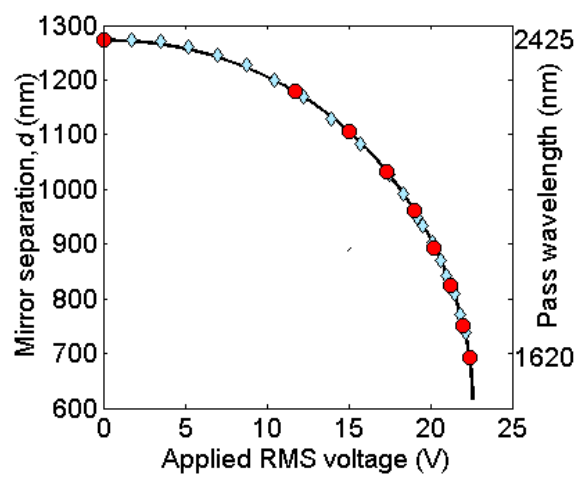

Fig 3. (a) filter tuning response of the current UWA microspectrometer and (b) Measured tuning response of spectrometer as a function of applied RMS voltage 
To demonstrate the array potential of these devices we have demonstrated some preliminary 2-D array implementations. Fig. 4 illustrates a preliminary array implementation of the microspectrometer. Note, however, this design incorporates the original design microspectrometer (i.e. pre 50\%-gap tuning), because it is easier to fabricate in an array form.

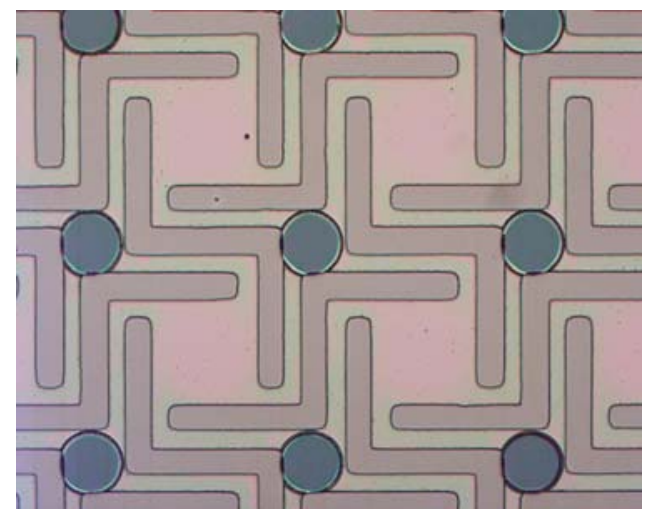

Fig 4. Preliminary demonstration of microspectrometers in array form.

\section{Other on-FPA applications - Fixed linear variable filters for multispectral imaging}

Although these devices technically don't have an electromechanical component (i.e. technically not MEMS), they are manufactured using the same technologies. Linear variable filters are a simple spectrometer technology, requiring no moving parts. Linear variable filters are useful in applications where a number of known fixed wavelengths are required, in a production-line environment. In this case, 2-D spectral imaging is not necessary because the production line provides the second spatial dimension temporally by translating the imaged object across the imaging array. For this application, we have proposed the use of a 2-D imaging array as shown in Fig. 5(a), using the second dimension to capture spectral information. In other words, a filter of a different wavelength is placed above each column of the 2-D imaging array. As the production line translates across the imaging array, each column of the array progressively images the object of interest. By time-shifting the data from each column, spectral data of that object can be combined into a single data set.

As an initial proof-of-concept device, we have fabricated a 1-D array as shown in Fig. 5(b). This device comprises a single row of the proposed device in Fig. 5(a). This consists of a linear array of fixed (non-tunable) Fabry Perot filters, fabricated optically ahead of an array of infrared photodiodes. The mirrors in this device are fabricated using the same germanium - silicon monoxide - germanium layer structure used in the tunable microspectrometer devices. The inter-mirror gap in the filters is created with an optically transparent silicon monoxide material of appropriate thickness.

To create a set of linear variable filers, the inter-mirror gap spacing is graded across the array. It is evident from Fig. 6 that the filters in the array produce a set of spectral transmission peaks at uniformly separated wavelengths. It is evident from the modeling and measurements that there is some scope for improvement of the actual devices, in terms of spectral resolution and peak transmission. However, the spectral peak locations can be very accurately controlled during fabrication.

\section{Conclusions}

We have demonstrated a MEMS microspectrometer technology with potential application to on-FPA implementation, to realize a spectrometer array on chip. This has many advantages over traditional imaging spectrometers, including significantly reduced size, weight and power requirements, as well as the ability reduce computational load by intelligently acquiring only the required spectral information of any given region of the FPA. These factors hold great promise for in airborne environments. This work has also demonstrated a linear variable array of filters with potential application, in 2-D array form, to process control in moving production line environments. 
(a)

(b)
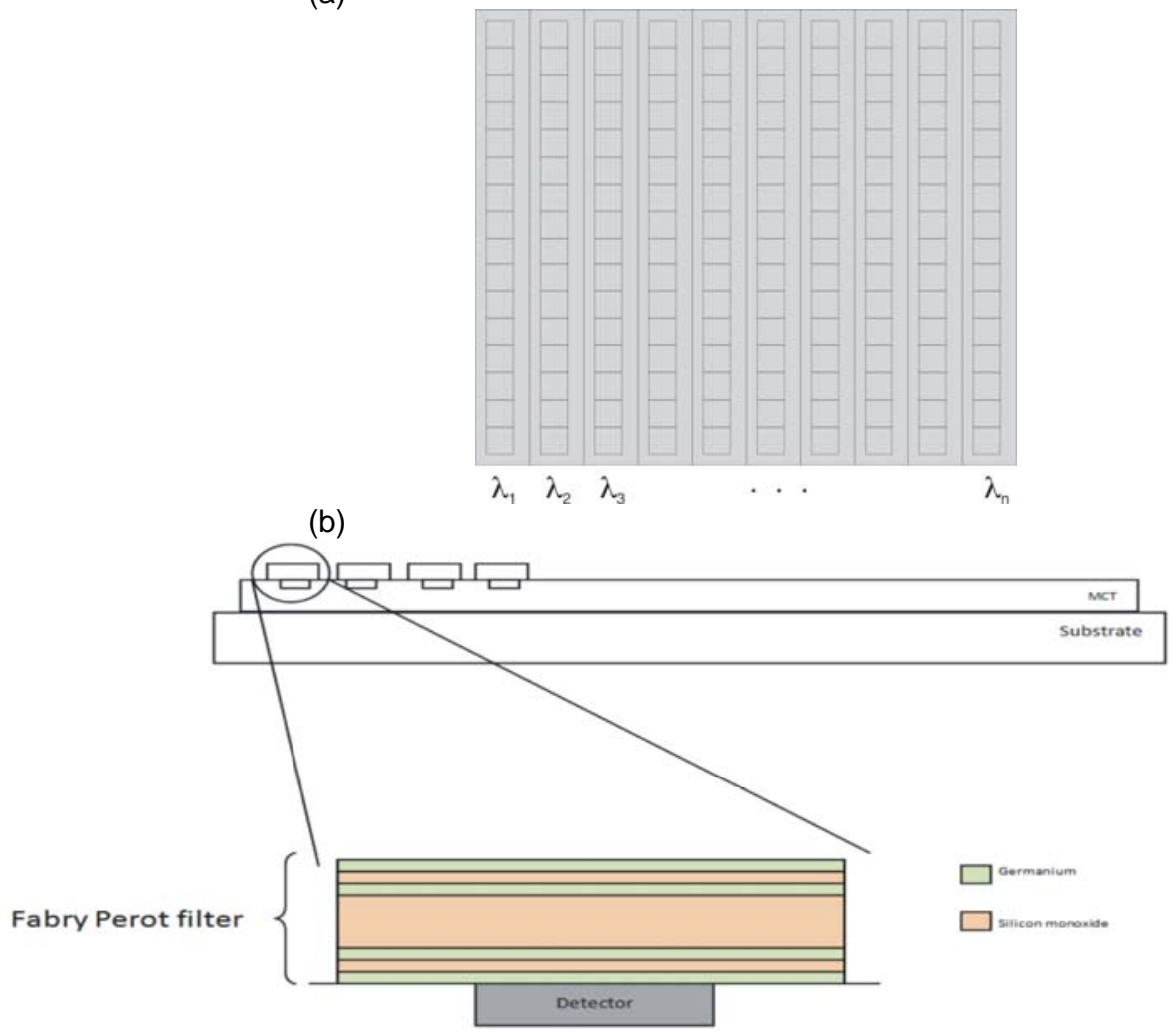

Fig 5. (a) Concept of Fixed linear variable array for imaging in a production line environment, (b) Design of a set of linear variable Fabry Perot filters

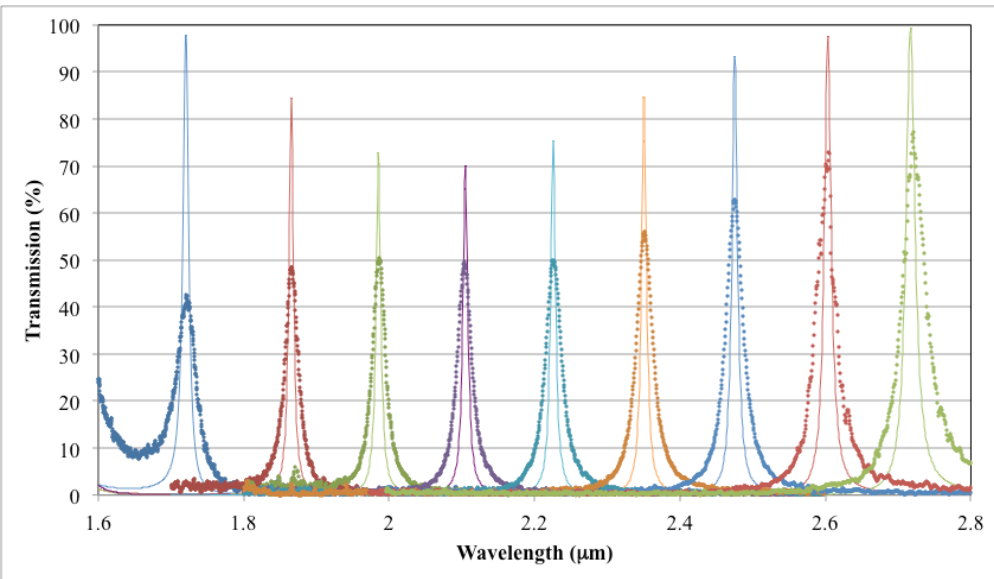

Fig 6. Modeled (lines) and measured (dots) performance of a fabricated set of linear variable Fabry Perot filters. 


\section{REFERENCES}

[1] Keating A. J., Antoszewski J., Silva K. K. M. B. D., Winchester K. J., Nguyen T., Dell J. M., Musca C. A, Faraone L., "Optical Performance of a MEMS Tunable IR Micro spectrometer", In Proceedings of COMMAD, pp $224-227,2006$

[2] Milne J. S., Dell J. M., Keating A. J., Faraone L., "Widely Tunable MEMS-Based Fabry-Perot Filter", J. MEMS, 18(4), pp $905-913,2009$.

[3] Keating A. J., Antoszewski J., Silva K. K. M. B. D., Winchester K. J., Nguyen T., Dell J. M., Musca C. A, Faraone L.,Mitra P., Beck J. D., Skokan M. R.,Robinson J. E., "Design and Characterization of Fabry-Perot MEMS-Based Short-Wave Infrared Microspectrometers", J. Elec. Mat., 37(12), pp. 1811 - 1820, 2008.

[4] P. S. Sumant, A. C. Cangellaris, and N. R. Aluru, Modeling of Dielectric Charging in RF MEMS Capacitive Switches, Microwave and Opt. Technol. Lett., v. 49, n. 12, pp 3188-3192, Dec 2007.

[5] Z. Peng, X Yuan, J. C. M. Hwang, D. I. Forehand, and C. L. Goldsmith, Dielectric Charging of RF MEMS Capacitive Switches under Bipolar Control-Voltage Waveforms, IEEE MTT-S Int. Microwave Symp. Dig., 18171820, 2007.

[6] H. Camon and C. Ganibal, Advantages of alternative actuating signal for MEMS actuators, Sensors and Actuators A, v. 136, pp 299-303, 2007. 